\title{
A universal TLR7-nanoparticle adjuvant promotes broad immune responses against heterologous strains of Influenza and SARS-CoV-2
}

\section{Qian Yin}

Stanford University

Wei Luo

Stanford University

Venkata Vamsee Aditya Mallajosyula

Stanford University https://orcid.org/0000-0002-0658-3652

Yang Bo

University of Illinois at Urbana, Champaign

Jing Guo

Stanford University

Jinghang Xie

Stanford University

Meng Sun

Stanford University

Rohit Verma

Stanford University

Christian Constantz

Stanford University

Lisa Wagar

Stanford University

Jing Li

Stanford University

\section{Elsa Sola}

Stanford University

Neha Gupta

Stanford University

Oliver Kask

Stanford University

Jianghong Rao

Stanford University https://orcid.org/0000-0002-5143-9529

Yueh-hsiu Chien 
Stanford University

Jianjun Cheng

University of Illinois at Urbana Champaign https://orcid.org/0000-0003-2561-9291

\section{Bali Pulendran}

Stanford University https://orcid.org/0000-0001-6517-4333

\section{Mark Davis ( $\square$ mmdavis@stanford.edu )}

Stanford University https://orcid.org/0000-0001-6868-657X

\section{Article}

Keywords: Influenza, SARS-CoV-2, TLR7-nanoparticle adjuvant

Posted Date: October 12th, 2021

DOl: https://doi.org/10.21203/rs.3.rs-936346/v1

License: (c) (1) This work is licensed under a Creative Commons Attribution 4.0 International License. Read Full License

Version of Record: A version of this preprint was published at Nature Materials on January 30th, 2023. See the published version at https://doi.org/10.1038/s41563-022-01464-2. 


\section{Abstract}

Fully effective vaccines for viruses such as Influenza and SARS-CoV-2 must elicit a diverse repertoire of antibodies against multiple drifted virus strains. However, how to achieve a diverse response has no general solution except to combine multiple strains, which risks diluting the response for all strains included. Here, we describe the synthesis of a universal, toll-like receptor 7 agonist (TLR7)-nanoparticle adjuvant, TLR7-NP, constructed from TLR7 agonist-initiated ring-opening polymerization of lactide and self-assembly with poly(ethylene glycol)- $b$-poly(lactic-co-glycolic acid). When mixed with Alum-adsorbed antigens, this TLR7-NP adjuvant elicited cross-reactive antibodies for both dominant and subdominant epitopes, as well as antigen-specific $\mathrm{CD} 8^{+} \mathrm{T}$ cell responses. TLR7-NPs adjuvanted influenza subunit vaccine successfully protected mice from heterologous viral challenge. TLR7-NPs also enhanced the antibody response to a SARS-CoV-2 subunit vaccine against multiple variants and revealed the mobilization of a virus-like response. We further demonstrate enhanced antigen-specific responses in human tonsil organoids with this novel adjuvant.

\section{Introduction}

Vaccines which mimic or are derived from infectious diseases represent the most efficient and costeffective means to control dangerous pathogens and preserve public health. Since their first use by Jenner in the late eighteenth century, vaccines have elicited potent immune responses against deadly infectious diseases and have saved countless lives ${ }^{1}$. Currently, vaccines against the severe acute respiratory syndrome coronavirus 2 (SARS-CoV-2) viruses were developed with unprecedented speed to power the global fight against this pandemic ${ }^{2}$. Yet, many challenges to vaccine development persist, and there have been many failures, and partial successes. Current influenza vaccines are among the latter, likely because they induce antibodies against the immunodominant part of virus - the globular head of hemagglutinin (HA). However, due to the immune selection pressure, the globular head of HA constantly undergoes antigenic drift, which leads to the manufactured influenza vaccines lacking cross-protection against drifted or shifted viral strains and requires them to be updated each year ${ }^{3}$. While not as variable as influenza, many variants of SARS-CoV-2 have been reported, and one of these, the delta variant, is much more infectious and is greatly complicating efforts to control the pandemic ${ }^{4,5}$. These variants of concern in general carry mutations in the spike protein, which through its receptor-binding domain (RBD) binds to the angiotensin-converting enzyme 2 (ACE2) receptor to enter cells, and all current vaccines target this interaction in order to prevent serious disease. Variants in the spike protein can lead to significantly reduced vaccine effectiveness ${ }^{6}$. As such, there is a critical need to develop broadly protective vaccines that can induce cross-reactive antibody responses against multiple drifted virus strains.

Compared to the immunodominant yet hypervariable head domain of HA, subdominant epitopes in the $\mathrm{HA}$ stem are more conserved and have the potential to generate cross-reactive immune response $\mathrm{e}^{7,8}$. Tremendous efforts have been focused on antigen design to improve humoral responses against these conserved yet subdominant epitopes, such as multivalent arrays of HA presented on the self-assembled 
protein nanoparticles 9 10, 'mosaic' nanoparticle immunogens with multiple related HA strains displayed

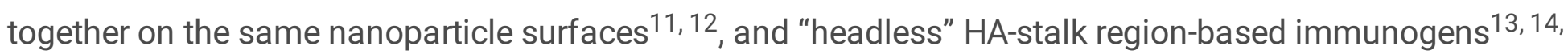
15, 16. Though effective, these approaches often involve sophisticated protein engineering processes, and the immunogens may not be rapidly and readily scalable for the global supply during the pandemic.

In addition to antigen, adjuvants plays a crucial role in enhancing, prolonging, and modulating vaccineinduced immune responses ${ }^{17}$. However, apart from a study ten years ago by Golding and colleagues ${ }^{18}$ that reported that MF59 adjuvant can broaden a flu response, finding adjuvants that can improve the diversity of a vaccine response has not been extensively studied ${ }^{19,20}$. Currently, aluminum hydroxide (alum) is the primary adjuvant used in commercial vaccines ${ }^{21}$. But even alum adjuvanted vaccines, especially for those using inactivated, subunit, or purified recombinant proteins, often require multiple doses to achieve protection and failed to induce broad antibody responses. ${ }^{17}$ Besides alum, a variety of toll-like receptors (TLRs) agonists have been explored as new adjuvant candidates ${ }^{22}$. For example, the synthetic TLR7 ligand imidazoquinoline and its derivatives have been shown to stimulate both innate and adaptive immunity by activating the nuclear factor kappa-light-chain enhancer of activated B cells (NFkB) pathway. Imiquimod, one example of this class, serving as the active component in the topical cream Aldara ${ }^{\circledR}$, is approved for human use to treat skin cancer and genital warts in the $1990 \mathrm{~s} .{ }^{23}$ Though a library of potent imidazoquinolines is under continuous development, their use as vaccine adjuvants has not progressed beyond clinical trials due to its rapid diffusion from the injection site and subsequently undesirable systemic immune activation ${ }^{24}$. To enhance the local adjuvant activity and minimize systemic diffusion, an adjuvant system using alum-adsorbed TLR7 agonist (trade name: AS37) ${ }^{25}$ has been developed and evaluated in clinical trials ${ }^{26}$. However, using it to enhance antibody breath hasn't been reported.

Here, we present an alternative TLR7-nanoparticle (TLR7-NP) adjuvant system to address the abovementioned issues. By uniquely integrating the TLR7 agonist-initiated ring-opening polymerization (ROP) chemical reaction into the formulation of NPs, we successfully demonstrated the quantitative incorporation of a synthetic TLR7 agonist into NPs with predefined drug loading and 100\% incorporation efficiency. The resulting biocompatible TLR7-NP adjuvant can be easily scaled up with wellcontrolled physicochemical properties including sub- $100 \mathrm{~nm}$ size, very narrow size distributions, and selfadjusted drug release kinetics - sustainable release during circulation and accelerated release upon internalization into cells. Compared to the alum-adsorbed TLR7 agonist (TLR7-Alum), TLR7-NP significantly improved in vivo retention, draining lymph node accumulation, and cellular uptake of this nanoparticle by various antigen-presenting cells (APCs), leading to the persistent mobilization and activation of dendritic cells and cells of monocytic lineage in draining lymph nodes. Critically, the TLR7NP significantly enhanced and extended the germinal center (GC) response, which is associated with an increased ratio of $\mathrm{T}$ follicular helper (Tfh) to regulatory follicular $\mathrm{T}$ cells $(\mathrm{Tfr})$. More surprisingly, this TLR7-NP also efficiently promoted CD8 T cell responses in draining lymph nodes and lung tissues, which 
are rarely seen in conventional vaccine-induced immune responses ${ }^{17}$. Importantly, the TLR7-NP induced significantly less systemic cytokine release compared to TLR7-Alum adjuvant.

We further demonstrate the broad applications of this TLR7-NP adjuvant system in multiple vaccines, including influenza vaccines and with a SARS-CoV-2 vaccine. Influenza HA adjuvanted with the TLR7-NP and efficiently induced early and high levels of cross-reactive antibody responses to HAs from multiple heterologous influenza strains. Remarkably, TLR7-NP adjuvanted vaccine induced early and markedly improved titers of antibodies binding to the conserved HA stem region, providing an avenue to developing the universal flu vaccine. As a result, this TLR7 agonist-NP adjuvanted influenza vaccine generated effective cross-protection against heterosubtypic influenza virus challenge. Furthermore, combining TLR7-NPs with full-length spike protein from SARS-CoV-2 outperformed a TLR7-Alum adjuvanted vaccines by inducing a high-level antibody response to spike protein and the RBD from multiple SARSCoV-2 variants, including B.1.1.7, B.1.351, P.1, and B.1.617 in mice. Most importantly, in combination with one clinically evaluated SARS-CoV-2 antigen, a TLR7-NP adjuvanted vaccine significantly promoted plasmablast (PB) differentiation and antibody isotype switching compared to antigen alone in a human tonsil organoid system, demonstrating the translational potential of TLR7-NPs as an adjuvant.

\section{Results}

\section{Synthesis and characterization of gardiquimod-polylactide nanoparticles (TLR7-NPs)}

The TLR7-NPs were made by co-nanoprecipitation of TLR7-PLA polymer conjugates and poly(ethylene glycol)-b-poly(lactic-co-glycolic acid) (PEG-PLGA) (Fig. 1a). TLR7-PLA polymer conjugates were synthesized by using gardiquimod, a potent agonist for TLR7 receptor expressed in both mouse and human, to initiate the ring-opening polymerization (ROP) of lactide $27,28,29$. This method allowed for quantitative incorporation of gardiquimod into PLA polymers and resulted in TLR7-PLA conjugates with precisely controlled composition and molecular weights (Fig. S1 and S2). At a monomer/initiator (LA/gardiquimod) ratio of 25 , gardiquimod loading was achieved as high as 14.8 wt $\%$ with nearly $100 \%$ incorporation efficiency. The resultant TLR7-PLA polymer conjugates were then mixed with PEG-PLGA in dimethylformamide (DMF) followed by nanoprecipitation into rapidly stirred water to self-assemble into TLR7-NPs with $77 \mathrm{~nm}$ hydrodynamic diameter and narrow size distributions (polydispersity index = 0.105) characterized by dynamic light scattering (DLS) (Fig. 1b).

In the design of TLR7-NPs, gardiquimod was conjugated to PLA polymer through ester linkages and could be released from NPs subjected to hydrolysis of ester bonds under physiological conditions. Released gardiquimod from TLR7-NPs (red) shared the identical UV-vis absorbance spectrum as the original gardiquimod compound (black), providing evidence of releasing unmodified TLR7 agonist without any residual chemical groups (Fig. 1C). To mimic the release profile of gardiquimod from TLR7NPs in the body, both in circulation and in endosomes/lysosomes, we next conduct the kinetic studies of 
TLR7-NPs at different pH 5.0 (endosomes/lysosomes) and 7.4 (circulation). Consistent with expectations, gardiquimod was released from NPs in a sustained manner without burst release effects, potentially minimizing the undesired systemic toxicity during the circulation. The release rate of gardiquimod from NPs was accelerated at increased acidities, likely due to faster hydrolysis at lower $\mathrm{pH}$, which is important for robust activation of the intracellular TLR7 receptors once NPs are internalized into cells (Fig. 1d).

\section{In vivo retention, lymph node trafficking and cellular internalization of TLR7-NPs}

To determine how TLR7-NPs influence the in vivo trafficking of gardiquimod, gardiquimod in the TLR7PLA was labeled with AlexaFluor647 (AF647) and co-nanoprecipitated with PEG-PLGA to formulate into NPs (Fig. S3). For comparison, we made gardiquimod adsorbed on alum, termed as TLR7-Alum, as previously reported ${ }^{25}$ and labeled gardiquimod with AF647 (Fig. S4). These two AF647-labeled adjuvants combined with alum-adsorbed OVA antigen were subcutaneously injected into C57BL/ 6 mice tail base, and the fluorescence of AF647 at the injection site was tracked by whole-body fluorescence imaging. TLR7-Alum was rapidly cleared from the injection site only 1 day post injection (p.i.), whereas AF647 labeled gardiquimod in the TLR7-NPs persisted for more than 3 days (Fig. 2a). Draining lymph nodes were dissected at days 1 and 3 post injection to evaluate the accumulation of gardiquimod through measuring the fluorescence intensity of AF647. As shown in Fig. 2b, the fluorescence intensity in the draining lymph nodes was significantly enhanced with TLR7-NPs as early as 1 day post injection, and the increased fluorescence intensity was maintained for as long as 3 days post immunization. Compared to TLR7-Alum, TLR7-NPs led to a 20-fold increase at day 1 and a 10-fold increase at day 3 in fluorescence signals in draining lymph nodes after immunization. These results suggest that TLR7-NPs were preferentially targeted and retained in the draining lymph nodes.

Given the preferential in vivo retention and enhanced LN targeting of TLR7-NPs, we next sought to evaluate how TLR7-NPs impact the cellular internalization of gardiquimod at a single-cell level using flow cytometry. Strikingly, there was a 5.5 to 27.8 -fold increase in the cellular uptake of TLR7-NPs in the vast majority of antigen-presenting cells (APCs) in draining lymph nodes, including B cells, dendritic cells, macrophages, and Ly $6 \mathrm{C}^{\text {hi }}$ inflammatory monocytes, compared to that of TLR7-Alum as early as 1 day post injection. This enhanced cellular uptake of TLR7-NPs in various APCs remained at a high level as long as 3 days post injection, with a 5.4 to 22.9-fold increase compared to TLR7-Alum (Fig. 2c). Interestingly, the persistent adjuvant capture of APCs led to potent and persistent mobilization of DCs and cells of monocytic lineage in draining lymph nodes (Fig. 2d and Fig. S5).

\section{TLR7-NPs potently boosts and prolongs both GC and extrafollicular B cell responses}

The enhanced and sustained activation of innate immune cells by TLR7-NPs encouraged us to assess the subsequent effect on adaptive immune responses. Vaccine efficacy largely depends on germinal center (GC) activity, which selects for high-affinity antibody producing B cells and the formation of durable B cell memory. To test whether TLR7-NPs as adjuvants might alter GC dynamics, two groups of 
C57BL/ 6 mice received an equivalent dose of NP-OVA as the antigen along with TLR7 agonist two different forms: one group was immunized with alum-adsorbed NP-OVA and TLR7-Alum and the other group with alum-adsorbed NP-OVA and TLR7-NPs. We then did a time course study from day 4 to day 22 post injection. As shown in Fig. 3a, GC B cells were detected starting at day 7 in draining lymph nodes, and their numbers were significantly higher in the TLR7-NP-treated group compared to TLR7-Alum group. The difference in GC B cell number between the two groups was maintained to day 22, the latest time point we have tested (Fig. 3a). Notably, despite decreasing GC B cell numbers after day 7, GC B cell numbers at day 22 in the TLR7-NP group were still higher than the peak numbers in the TLR7-Alum group. GC selection relies on B-T cell interaction, in which T cells provide essential help to support GC B cells survival and selection ${ }^{30}$. Consistent with GC B cell kinetics, follicular T cells expressing CXCR5 and PD1 were also highly expanded in TLR7-NP group. Follicular T cells contain T follicular helper cells (Tfh) and $T$ follicular regulatory cells ( $\mathrm{Tfr}$ ). Tfh are the actual helper cells that promote $\mathrm{GC}$ responses, whereas $\mathrm{Tfr}$ express regulatory $T$ cell marker FOXP3 and function to restrain GC reaction. To characterize the composition, we first gated on total follicular T cells $\left(\mathrm{BCL}^{+}{ }^{+} \mathrm{PD} 1^{+}\right)$and further gated $\mathrm{Tfr}$ subset based on FOXP3 expression (Fig. 3b). Strikingly, there were 30-40\% follicular T cells on day 7 post immunization from TLR7-Alum group expressing FoxP3 (defined as Tfr) (Fig. 3b). In TLR7-NP group, the percentage of Tfr dropped significantly to $~ 10 \%$. All these data strongly suggest the nanoparticle adjuvant formulation is very potent in promoting GC response. Interestingly, TLR7-NPs could also promote an extrafollicular B cell response with a markedly increased number of early plasmablasts in TLR7-NP group compared to TLR7-Alum group (Fig. 3a). With these drastic changes in B cell characteristics, we found that TLR7-NPs were not only enhanced the magnitude of antibody secretion, but also altered the quality of antibody responses. All mice immunized with TLR7-NPs adjuvanted NP-OVA, in which contains immunodominant epitope NP and subdominant epitope OVA, generated high levels of antibodies against the subdominant OVA epitope. In contrast, $83.3 \%$ (5 out of 6 ) of mice immunized with TLR7-Alum adjuvanted NP-OVA couldn't (Fig. 3c).

\section{TLR7-NPs adjuvanted vaccine elicits a robust antigen-specific CD8 ${ }^{+} \mathrm{T}$ cell response}

TLR7-NPs have efficiently induced persistent mobilization and activation of conventional type 1 dendritic cells ( $C D C 1$ ), which are vital for cross-priming $C D 8^{+} T$ cells. This, we sought to determine if TLR7-NPs adjuvant could also enhance antigen-specific $\mathrm{CD} 8^{+} \mathrm{T}$ cell responses compared to other immunization strategies, which generally poorly stimulate $C D 8^{+} T$ cells. As shown in Fig. 3d, there was a marked enhancement in the number of effector memory (CD44 $\left.{ }^{\text {hi }} \mathrm{CD} 62 \mathrm{~L}^{\text {low }}\right)$ OVA-specific CD ${ }^{+} \mathrm{T}$ cells in draining lymph nodes 7- and 14-days post immunization of TLR7-NPs adjuvanted vaccines (Fig. 3d). The proportion of $\mathrm{CD} 69^{\text {hi }} \mathrm{CD} 8^{+} \mathrm{T}$ cells rose significantly at 4 days post immunization and persisted until 14 days post immunization, indicating the early and persistent activation of OVA-specific $\mathrm{CD} 8^{+} \mathrm{T}$ cells in response to TLR7-NPs adjuvanted vaccination (Fig. 3d). Further, flow cytometry analysis showed TLR7NPs adjuvanted vaccine efficiently induced Granzyme B (Gzmb) producing $C D 8^{+} T$ cells in draining lymph nodes at 7 days post immunization. In contrast, the TLR7-Alum adjuvanted vaccine failed to induce 
Gzmb producing $\mathrm{CD} 8^{+} \mathrm{T}$ cells, demonstrating TLR7-NPs augment not only the quantity but also the quality of cellular immune responses (Fig. 3d).

As $\mathrm{CD} 8^{+} \mathrm{T}$ cells-mediated killing of intracellular pathogen-infected cells is critical for controlling viral replication in the lung, we next analyzed the $C D 8^{+} T$ cell responses in the lungs after 14 days post immunization. As shown in Fig. 3e, a large number of functional $\mathrm{CD} 8^{+} \mathrm{T}$ cells expressing Gzmb were observed in the lungs after receiving TLR7-NPs adjuvanted NP-OVA vaccine, whereas Gzmb producing $\mathrm{CD}^{+} \mathrm{T}$ cells were barely detected in the lungs of mice receiving TLR7-Alum adjuvanted vaccines (Fig. 3e). Altogether, TLR7-NPs were superior to TLR-Alum in inducing potent CD8 ${ }^{+}$T-cell responses.

\section{TLR7-NPs adjuvanted HA immunization induces cross-reactive and stem specific antibodies}

Development of a broadly cross-reactive universal influenza vaccine for clinical use has been challenging and thus far, unsuccessful. The primary reason is immunodominance, in which antibody and memory responses are focused on dominant epitopes, which leads to selection pressure and frequent viral escape mutations. The potent effect of TLR7-NP on promoting GC responses suggests their potential to tackle this issue. More importantly, TLR7-NPs adjuvant can drastically increase the ratio of Tfh to $\mathrm{Tfr}$, which might increase the diversity of B cell clones in the GC by providing additional T help. As such, we hypothesized that TLR7-NPs adjuvant might help overcome immunodominance and increase the breadth of antibody response against influenza HA. First, we performed the immunofluorescence staining of the draining lymph nodes from mice who received the immunization of HA plus TLR7-NPs and confirmed more GCs were observed at day 10 post immunization (Fig. 4a). Next, to test whether TLR7-NPs can increase the breadth of the response, we immunized mice with $\mathrm{H} 3 \mathrm{HA}$ from influenza strain $\mathrm{A} / \mathrm{Hong}$ Kong/1/1968 (HK68), a representative strain from the group 2 influenza viruses, and did serology for antibodies against HAs from heterologous strains at week 2 post primary immunization (Fig. 4b). The result showed that TLR7-NPs not only increased antibody titers against the immunized H3 HA from HK68, but also enhanced antibody responses against HA from another H3N2 strain, A/Hong Kong/4801/2014 (HK14). More importantly, 80\% of mice immunized with TLR7-NPs adjuvanted H3 HA were also able to make antibodies against HA from H7N9 (strain SH13), an influenza virus variant from a different subtype. In contrast, these heterosubtypic antibodies could not be detected in the mice immunized with HA adjuvanted by TLR7-Alum. To test whether TLR7-NPs could induce HA-specific antibodies against group 1 viruses as well, we used H1 HA from PR8 in a similar immunization experiment setting. Again, we found that mice immunized with TLR7-NPs adjuvanted PR8 HA generated significantly higher levels of antibodies against HA of cross-subtype or even cross-group (Fig. 4c). The head region of HAs from different influenza strains is highly variable and mutable, while the stem region is more conserved. We hypothesized that antibodies against stem might explain such cross-reactivity. To test if TLR7-NPs could skew the immunodominance from mutable HA head region to conserved stem region, we did ELISA with a headless HA protein derived from PR8 HA. We found that TLR7-NPs adjuvanted PR8 HA immunization did induce a much greater level of antibodies binding to the stem region (Fig. 4c). Next, we immunized mice with $\mathrm{H} 1 \mathrm{HA}$ from another strain (NC99) and tested for antibodies against headless and intact HA 
from PR8. With the change of immunogen to NC99 H1 HA, TLR-NPs still could enhance the antibody response towards stem region as well as intact HA from PR8 (Fig. 4d), confirming that the stem region is indeed conserved and targeted by cross-reactive antibodies. Hence, using three different HAs as the immunogen, we demonstrated the potential of TLR7-NPs as an adjuvant to boost cross-reactive antibody responses.

\section{TLR7-NPs adjuvanted HA immunization induces broad protection against heterosubtypic influenza viruses}

Given the potent cross-reactive antibody responses induced by TLR7-NP assisted vaccination, we reasoned that this adjuvant might help induce cross-protection against different influenza viruses. To evaluate this possibility, we immunized two groups of C57BL/ 6 mice with an equivalent dose of alumadsorbed NC99 HA as the antigen along with TLR7 agonist in TLR7-Alum or TLR7-NPs, at week 0 and week 3. Four weeks post boost; mice were challenged with a lethal dose of distinct PR8 H1N1 virus (Fig. 4e). In line with the observation of enhanced cross-reactivity with TLR7-NP adjuvantation (Fig. 4d), mice receiving the TLR7-NPs adjuvanted NC99 HA lost significantly less body weight upon challenge compared to mice receiving TLR7-Alum adjuvanted vaccine (Fig. 4f). Most of the mice that received TLR7-Alum adjuvanted NC99 HA failed to develop cross-protection against PR8 H1N1 viral challenge as only $\sim 40 \%$ of mice from this group survived the challenge (Fig. $4 \mathrm{~g}$ ). In contrast, more than $92 \%$ of mice immunized with TLR7-NP adjuvanted HA fully recovered after the infection (Fig. 4g). Furthermore, we performed a histologic analysis of lung sections from mice survivors 14 days post PR8 H1N1 viral infection. As illustrated in Fig. 4h, severe pathologic peribronchiolar inflammation associated with diffuse damage of structures were readily identified within the lung tissues of unvaccinated mice and mice who received TLR7-Alum adjuvanted vaccines. In contrast, TLR7-NPs adjuvanted HA immunization fully protected the mice from the infection induced pulmonary damage, maintaining similar architecture of lung tissues compared to uninfected mice (Fig. 4h). Combined, these results strongly demonstrate that a TLR7-NPs adjuvanted HA vaccine not induced cross-reactive antibodies but also offered strong cross protection against heterologous influenza infection.

\section{TLR7-NPs adjuvanted spike immunization induces cross-reactive antibodies against the SARS-CoV-2 variants}

The emergence of several SARS-CoV-2 variants with multiple amino acid changes in the Spike (S) protein and especially within the RBD has implications for the future control of the COVID-19 pandemic. Several of these mutations directly affect ACE2 receptor binding affinity, impacting infectivity, viral load, or transmissibility. Variants of concern include the Alpha variant United Kingdom (U.K.) 501Y.V1 lineage B.1.1.7, the Beta variant South Africa (S.A.) 501Y.V2 lineage B.1.351, the Gamma variant Brazil (B.R.) 501Y.V3 lineage P.1, and the Delta variant India B.1.617.2. To test if a TLR7-NPs adjuvant could increase the breadth of the antibody response against these variants, we immunized C57BL/ 6 mice with the fulllength spike protein from SARS-CoV-2 virus with TLR7-Alum or TLR7-NPs as an adjuvant (Fig. 5a) and characterized the antibody responses against the wildtype RBD, spike and S1 protein, as well as RBD and 
S1 protein from several variants of concern (Fig. 5b and 5c). After two immunizations, all mice immunized with TLR7-NP adjuvanted spike protein (Wuhan reference strain) developed high titers of antibodies against RBD with 501Y mutation and RBD of P.1 and B.1.617 variant, as well as S1 protein from B.1.1.7 and B.1.351 variants. In contrast, the mice immunized with TLR7-Alum adjuvanted spike had significantly lower antibody responses against immunized spike protein. Only 50-60\% of them developed detectable antibody responses against these variants. Similarly, for the Indian Delta variant (B.1.617.2), a highly contagious SARS-CoV-2 virus strain now circulating globally, TLR7-NPs were able to induce high titers of antibodies against its RBD domain in $89 \%$ of vaccinated mice, whereas only $50 \%$ of mice immunized with TLR7-Alum adjuvanted spike generated 1-2 log fold lower antibody titers (Fig. 5c).

\section{TLR7-NPs adjuvanted spike protein subunit vaccine elicits B cell differentiation and antibody response in 3D human tonsil organoid cultures}

To further investigate the translational potential of TLR7-NPs as an adjuvant for both mouses and human analyses, we used our recently developed human tonsil organoid system ${ }^{31}$ to assess the effects of TLR7-NPs when combined with the full-length SARS-CoV-2 spike protein. Organoid cultures were prepared and stimulated with spike protein with or without TLR7-NPs. After 14 days in culture, we assessed the phenotype changes of B cells in the culture and antibody specificities against SARS-CoV-2 spike in the supernatants. As shown in Fig. $6 \mathrm{a}$ and Fig. $6 \mathrm{~b}$, we observed a significant increase in Pre-GC B cells, GC-B cells and plasmablast differentiation in a subset of donors upon stimulation of Spike with TLR7-NPs compared to Spike protein only controls. Spike-specific IgM and IgA antibodies increased in 5 out of 6 donors when stimulating with Spike + TLR7-NPs compared to Spike alone (Fig. 6c). In addition, we also tested the adjuvanticity of TLR7-NPs in combination with a subunit vaccine currently under clinical testing, comprised of the SARS-CoV-2 spike protein RBD displayed on a I53-50 protein nanoparticle scaffold (termed as RBD-NP) ${ }^{32}$ in this organoid system. We performed a time-course singlecell RNA sequencing (scRNA-seq) study using the BD Rhapsody platform for sorted B cells from unadjuvanted RBD-NP cultures and RBD-NP + TLR7-NPs stimulated tonsil organoid cultures. We first examined $B$ cells states within our single-cell transcriptomic atlas and identified 8 distinct $B$ cell populations by uniform manifold approximation and projection (UMAP) based on their gene expression profile (Fig. 6d). As shown in Fig. 6e, both the frequencies and the numbers of GC B and plasmablasts (PB) notably increased as early as 4 days' post stimulation with the RBD-NP plus TLR7-NPs compared to RBD-NP alone. This is well in line with our previous observation that TLR7-NPs induced the early promotion of both extrafollicular responses and GC responses in the mouse (Fig. 3). More importantly, IgM PB, IgA PB, and IgG PB were all significantly increased in RBD-NP plus TLR7-NPs stimulated cultures, indicating the broad effects of TLR7-NPs on the antibody isotype switching of $B$ cells in human tonsil organoids (Fig. 6f). To further understand TLR7-NP-mediated signaling pathways on B cell differentiation, we then compared gene expression within B cells in both RBD-NP alone stimulated- and RBD-NP + TLR7NPs stimulated cultures. Gene Ontology (GO) analysis showed that genes associated with responses to viruses and type 1 interferon are remarkedly upregulated on 4 -day-old tonsil cultures stimulated with RBD-NP plus TLR7-NPs compared to RBD-NP alone (Fig. 6g). 


\section{TLR7-NPs adjuvant minimizes systemic immune toxicity}

As possible adjuvant mediated toxicity is an important consideration, we next evaluated this possibility by subcutaneously injecting the TLR7-NP adjuvanted vaccine into C57BL/ 6 mice, using TLR7-Alum as a control. TLR7-Alum rapidly diffused into circulation within 3 hours after a single injection, leading to acute inflammation, characterized by multiple elevated serum inflammatory cytokines including MCP-1, MIP1B, IFNA, IL-6, IP-10, and GROA (Fig. S6a, left). Among them, GROA and IL-6 maintained at a high level in the serum as long as 24 hours post immunization (Fig. S6a, right). We further evaluated the long-term toxicity by clinical chemistry at day 1 , day4, and day7 post immunization. The result showed the significantly increased blood urea nitrogen (BUN) enzyme level in TLR7-Alum immunized group at day1, indicating the acute kidney toxicities, presumably caused by fast renal clearance of small molecular TLR7 agonist in this formulation (Fig. S6b). In contrast, TLR7-NPs adjuvanted vaccines showed negligible systemic inflammation and renal toxicity.

\section{Discussion}

The immune response to vaccination is a function of a given formulation's spatiotemporal profile, with the timing and localization of antigen and danger signals dictating qualitative and quantitative aspects of vaccine-induced immunity. ${ }^{33}$ Herein we report a general strategy to enhance the breadth and magnitude of antibody responses elicited by vaccination, simply by controlling the adjuvants' physicochemical properties to enhance their in vivo performance and obviate toxicity. In the data shown here, in the context of TLR7 agonist-based adjuvant design, nanoparticulate formulation outperformed the alum adsorption, greatly improving their biological performance, and subsequently broadening protective responses against heterologous influenza virus strains. As such, this broadly applicable TLR7NP adjuvant platform holds the great potential for developing future universal vaccines against influenza virus or coronavirus to markedly increase our pandemic preparedness.

The induction of antibody breadth is usually associated with overcoming immunodominance. Germinal centers (GCs) as the sites of antibody diversification and affinity maturation are vitally important in shaping B cell responses, especially to novel antigenic challenges. Immunodominance in B cell response results from a few dominant clones outcompeting other clones in the GCs to produce plasma cells and to become long-lived memory B cells. Though the factors affecting immunodominance in the GC B cell response are not clear, previous studies have suggested that epitope accessibility, precursor frequency,

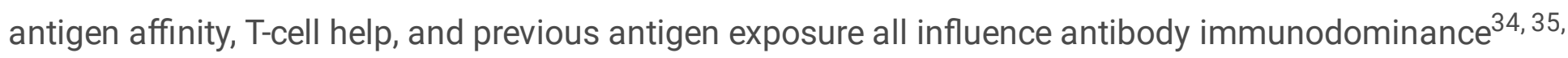
${ }^{36}$. Specifically, B cells in the GCs have to constantly compete for T-cell signals for selection and differentiation ${ }^{37}$. It has been reported that Tfh response is associated with HIV neutralizing antibody generation ${ }^{38,39}$ and $\mathrm{Tfr}$ can restrict the early GC response to control memory formation ${ }^{40}$. As shown in our studies, the significantly increased ratio of Tfh/Tfr in mice upon TLR7-NPs immunization might enhance T cell help to promote the survival of subdominant B cell clones in the GCs. In addition, TLR7NPs, in contrast to TLR7-Alum, drastically increase early extrafollicular plasmablast differentiation. The 
antibodies produced by these cells might impact the quality of GC responses by forming immune complexes with antigens to better stimulate GC B cells or promote Tfh differentiation.

We also demonstrate here that the nanoparticulate formulation of the TLR7 agonist greatly enhances the cellular immune responses needed for a robust vaccination response, which is well in line with the pioneering work by Seder et al. ${ }^{41}$ wherein protein antigens linked with TLR7/8-polymer conjugates to form particulates. These vaccine particulates elicit a significantly enhanced $\mathrm{CD} 8^{+} \mathrm{T}$ cell response and a direct correlation between hydrodynamic radii and magnitude of lymph node IL-12 production were observed. Though efforts toward vaccine development often focus on inducing effective antibody responses, eliciting both humoral and cellular immune responses can be superior, particularly when antibody titers are below the threshold required for protection. Notably, TLR7-NPs presented in this study generates high quality and magnitude of $\mathrm{CD} 8^{+} \mathrm{T}$ cell responses, which are not optimally induced by the most commonly used adjuvants in vaccines approved for human use, including alum and oil-in-water emulsion-based adjuvants. The TLR7-NPs reported herein could potentially accelerate the development of vaccines against pathogens that are controlled by cellular immune responses, including that causing malaria, tuberculosis, and leishmaniasis.

One considerable hurdle of vaccine adjuvant development is eliciting a sufficiently potent immune response while meeting the exceptional safety standard requirement for administration to healthy populations. Compared with alum adsorbed TLR7 agonists, the use of nanoparticles enables the spatiotemporal delivery of this small molecular agent to the draining lymph nodes, which could potentially enhance the "priming" of the immune system while reducing the likelihood of adverse side effects. Despite these advantages, the clinical translation of nanoparticle-based therapies has proven difficult. One such barrier is the difficulty in consistently manufacturing nanomedicines with optimal physicochemical properties. To our knowledge, the work described here represents the first use of a TLR7 agonist as the drug initiator in the ROP polymerization technique to construct TLR7-polymer conjugates with predefined drug loading and a uniform structure. The generated sub-100nm nanoparticles with its very narrow size distribution address challenges of sterile filtration using pore membranes below $0.2 \mathrm{um}$, avoiding the issues of particle clogging and the material loss observed by many other nanoparticle vaccine technologies whose sizes exceed the membrane pore size. Moreover, the formulated TLR7-NPs are biocompatible and biodegradable, which is important for their potential clinical translation.

In conclusion, the results presented here show how the adjuvant formulation can be optimized to enhance the magnitude and breadth of antibody responses while addressing some of the many challenges for translational applications. Most importantly, this TLR7-NP adjuvant has enormous potential as a tool for understanding the complexities of immune regulation and may help lead to broader and more effective influenza virus or SARS-CoV-2 vaccines.

\section{Declarations}

\section{Acknowledgments}


We thank X. Ji and M.Miranda for single -cell RNA-seq help, and the Stanford Nano Shared Facilities (SNSF), the Stanford Shared FACS Facility and The Human Immune Monitoring Center at Stanford for assistance in this study, and members of Davis laboratory for helpful advice and discussion. Funding: We are grateful for the support of the Howard Hughes Medical Institute (to M.M.D.), The Bill and Melinda Gates Foundation Pilot grant (to M.M.D.). The authors also thank the participants for donating their tissues for the tonsil organoids study.

\section{Author Contributions}

Q.Y., W.L., B.P. and M.M.D. conceived the project and guided it throughout. Q.Y., W.L., Y.B., J.G. J.X. M.S.

R.V. C.C. L.W. performed the experiments or data analysis. V.M. designed and produced antigen. E.S. and N.G assisted with human tonsil tissue processing. J.R., Y-H. C., and J.C. contribute to conceptual design of the study. Q.Y., W.L., and M.M.D. wrote the paper.

\section{Competing Interest Statement}

Q.Y., M.M.D., W.L., and B.P. are inventors on a patent application that describes the use of this nanoparticle for vaccine adjuvant.

\section{References}

1. Pollard, A.J. \& Bijker, E.M. A guide to vaccinology: from basic principles to new developments. Nat Rev Immunol 21, 83-100 (2021).

2. Krammer, F. SARS-CoV-2 vaccines in development. Nature 586, 516-527 (2020).

3. Innis, B.L. et al. Meeting report: Convening on the influenza human viral challenge model for universal influenza vaccines, Part 1: Value; challenge virus selection; regulatory, industry and ethical considerations; increasing standardization, access and capacity. Vaccine 37, 4823-4829 (2019).

4. Mlcochova, P. et al. SARS-CoV-2 B.1.617.2 Delta variant replication and immune evasion. Nature (2021).

5. Planas, D. et al. Reduced sensitivity of SARS-CoV-2 variant Delta to antibody neutralization. Nature 596, 276-280 (2021).

6. Abdool Karim, S.S. \& de Oliveira, T. New SARS-CoV-2 Variants - Clinical, Public Health, and Vaccine Implications. N Engl J Med 384, 1866-1868 (2021).

7. Ellebedy, A.H. et al. Induction of broadly cross-reactive antibody responses to the influenza HA stem region following H5N1 vaccination in humans. Proc Natl Acad Sci U S A 111, 13133-13138 (2014).

8. Tan, H.X. et al. Subdominance and poor intrinsic immunogenicity limit humoral immunity targeting influenza HA stem. J Clin Invest 129, 850-862 (2019).

9. Lopez-Sagaseta, J., Malito, E., Rappuoli, R. \& Bottomley, M.J. Self-assembling protein nanoparticles in the design of vaccines. Comput Struct Biotechnol J 14, 58-68 (2016).

10. Haynes, J.R. Influenza virus-like particle vaccines. Expert Rev Vaccines 8, 435-445 (2009). 
11. Schwartzman, L.M. et al. An Intranasal Virus-Like Particle Vaccine Broadly Protects Mice from Multiple Subtypes of Influenza A Virus. mBio 6, e01044 (2015).

12. Boyoglu-Barnum, S. et al. Quadrivalent influenza nanoparticle vaccines induce broad protection. Nature 592, 623-628 (2021).

13. Yassine, H.M. et al. Hemagglutinin-stem nanoparticles generate heterosubtypic influenza protection. Nat Med 21, 1065-1070 (2015).

14. Impagliazzo, A. et al. A stable trimeric influenza hemagglutinin stem as a broadly protective immunogen. Science 349, 1301-1306 (2015).

15. Steel, J. et al. Influenza virus vaccine based on the conserved hemagglutinin stalk domain. mBio 1 (2010).

16. Mallajosyula, V.V. et al. Influenza hemagglutinin stem-fragment immunogen elicits broadly neutralizing antibodies and confers heterologous protection. Proc Natl Acad Sci U S A 111, E25142523 (2014).

17. Reed, S.G., Orr, M.T. \& Fox, C.B. Key roles of adjuvants in modern vaccines. Nat Med 19, 1597-1608 (2013).

18. Khurana, S. et al. MF59 adjuvant enhances diversity and affinity of antibody-mediated immune response to pandemic influenza vaccines. Sci Trans/ Med 3, 85ra48 (2011).

19. Kim, J.H., Davis, W.G., Sambhara, S. \& Jacob, J. Strategies to alleviate original antigenic sin responses to influenza viruses. Proc Natl Acad Sci U S A 109, 13751-13756 (2012).

20. Hong, D.K. et al. Cationic lipid/DNA complex-adjuvanted influenza A virus vaccination induces robust cross-protective immunity. J Viro/ 84, 12691-12702 (2010).

21. He, P., Zou, Y. \& Hu, Z. Advances in aluminum hydroxide-based adjuvant research and its mechanism. Hum Vaccin Immunother 11, 477-488 (2015).

22. Maisonneuve, C., Bertholet, S., Philpott, D.J. \& De Gregorio, E. Unleashing the potential of NOD- and Toll-like agonists as vaccine adjuvants. Proc Natl Acad Sci U S A 111, 12294-12299 (2014).

23. Bhagchandani, S., Johnson, J.A. \& Irvine, D.J. Evolution of Toll-like receptor $7 / 8$ agonist therapeutics and their delivery approaches: From antiviral formulations to vaccine adjuvants. Adv Drug Deliv Rev 175,113803 (2021).

24. Vasilakos, J.P. \& Tomai, M.A. The use of Toll-like receptor $7 / 8$ agonists as vaccine adjuvants. Expert Rev Vaccines 12, 809-819 (2013).

25. Fox, C.B. et al. Adsorption of a synthetic TLR7/8 ligand to aluminum oxyhydroxide for enhanced vaccine adjuvant activity: A formulation approach. J Control Release 244, 98-107 (2016).

26. Gonzalez-Lopez, A. et al. Adjuvant effect of TLR7 agonist adsorbed on aluminum hydroxide (AS37): A phase I randomized, dose escalation study of an AS37-adjuvanted meningococcal C conjugated vaccine. Clin Immunol 209, 108275 (2019).

27. Yin, Q. et al. Pamidronate functionalized nanoconjugates for targeted therapy of focal skeletal malignant osteolysis. Proc Natl Acad Sci U S A 113, E4601-4609 (2016). 
28. Yin, Q. et al. Drug-initiated ring-opening polymerization of O-carboxyanhydrides for the preparation of anticancer drug-poly(0-carboxyanhydride) nanoconjugates. Biomacromolecules 14, 920-929 (2013).

29. Yin, Q., Yin, L., Wang, H. \& Cheng, J. Synthesis and biomedical applications of functional poly(alphahydroxy acids) via ring-opening polymerization of O-carboxyanhydrides. Acc Chem Res 48, 17771787 (2015).

30. Shulman, Z. et al. Dynamic signaling by $T$ follicular helper cells during germinal center $B$ cell selection. Science 345, 1058-1062 (2014).

31. Wagar, L.E. et al. Modeling human adaptive immune responses with tonsil organoids. Nat Med 27, 125-135 (2021).

32. Walls, A.C. et al. Elicitation of Potent Neutralizing Antibody Responses by Designed Protein Nanoparticle Vaccines for SARS-CoV-2. Cell 183, 1367-1382 e1317 (2020).

33. Irvine, D.J., Swartz, M.A. \& Szeto, G.L. Engineering synthetic vaccines using cues from natural immunity. Nat Mater 12, 978-990 (2013).

34. Abbott, R.K. \& Crotty, S. Factors in B cell competition and immunodominance. Immunol Rev 296, 120-131 (2020).

35. Akram, A. \& Inman, R.D. Immunodominance: a pivotal principle in host response to viral infections. Clin Immuno/ 143, 99-115 (2012).

36. Abbott, R.K. et al. Precursor Frequency and Affinity Determine B Cell Competitive Fitness in Germinal Centers, Tested with Germline-Targeting HIV Vaccine Immunogens. Immunity 48, 133-146 e136 (2018).

37. Shlomchik, M.J., Luo, W. \& Weisel, F. Linking signaling and selection in the germinal center. Immunol Rev 288, 49-63 (2019).

38. Swathirajan, C.R. et al. Association of circulatory Tfh-like cells with neutralizing antibody responses among chronic HIV-1 subtype C infected long-term nonprogressors and progressors. Pathog Dis 77 (2019).

39. Havenar-Daughton, C., Lee, J.H. \& Crotty, S. Tfh cells and HIV bnAbs, an immunodominance model of the HIV neutralizing antibody generation problem. Immunol Rev 275, 49-61 (2017).

40. Clement, R.L. et al. Follicular regulatory T cells control humoral and allergic immunity by restraining early B cell responses. Nat Immuno/ 20, 1360-1371 (2019).

41. Lynn, G.M. et al. In vivo characterization of the physicochemical properties of polymer-linked TLR agonists that enhance vaccine immunogenicity. Nat Biotechno/ 33, 1201-1210 (2015).

\section{Figures}


Figure 1:

a

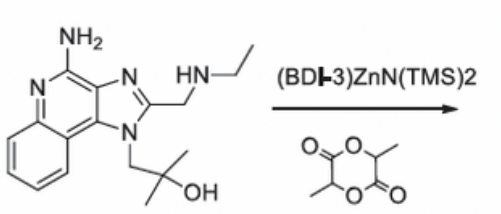

Initiator: gardiquimod

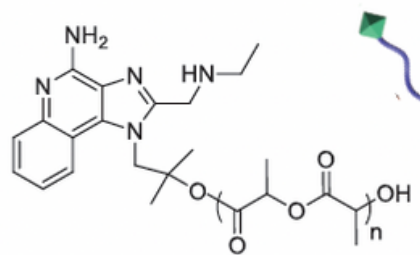

Gardiquimod-polylactide (TLR7-PLA)

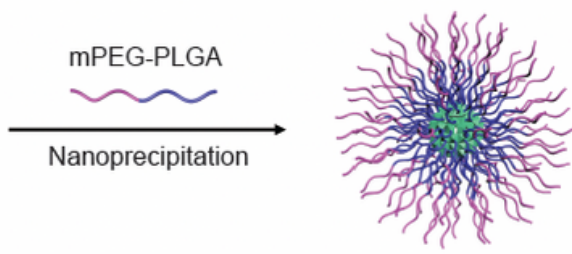

TLR7-PLA NP b

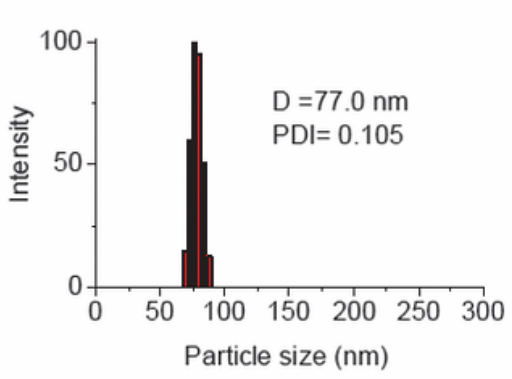

c

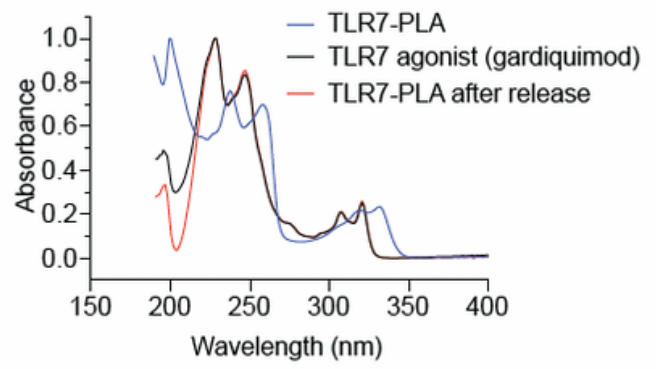

d $\rightarrow \mathrm{pH}=7$

$-\mathrm{pH}=5$

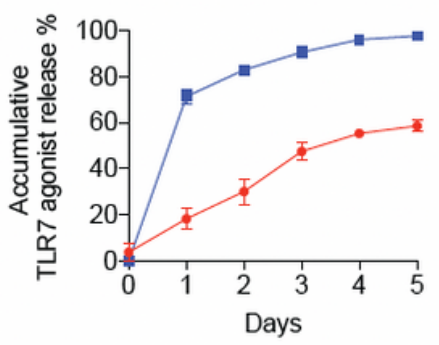

\section{Figure 1}

Synthesis, formulation, and characterization of gardiquimod-polylactide nanoparticles (TLR7-NPs). (a) Schematic illustration of synthesizing TLR7-PLA polymer conjugate via gardiquimod initiated ringopening polymerization of lactide and preparing TLR7-NPs through nanoprecipitation. (b) Hydrodynamic sizes of TLR7-NPs characterized by DLS analysis. (c) The UV absorbance of TLR7-PLA polymer, TLR7 agonist (gardiquimod), and TLR7-NPs after release measured by UV spectrometer at $\lambda=321 \mathrm{~nm}$. (d) Release kinetic profile of TLR7 agonist (gardiquimod) from TLR7-NPs in PBS buffer at pH 5.0 and pH 7.4. 
Figure 2:

a
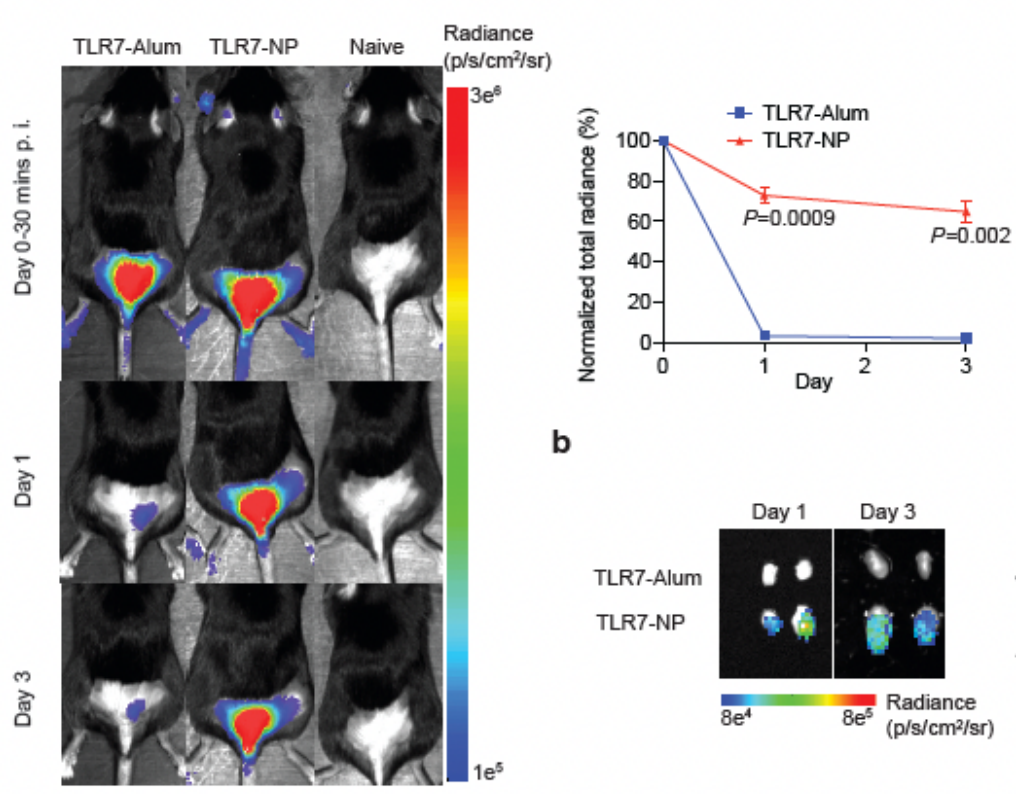

b

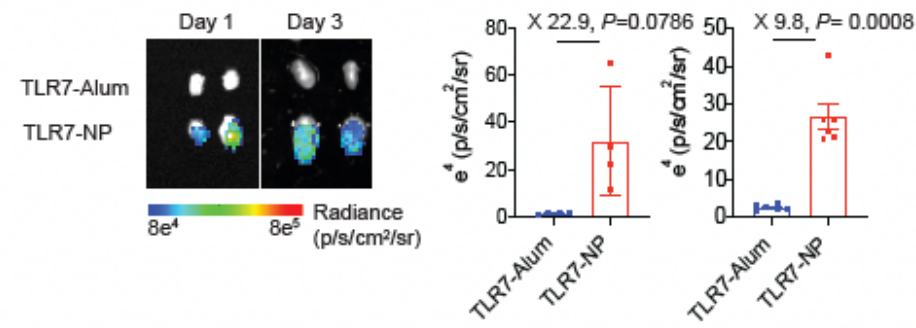

C

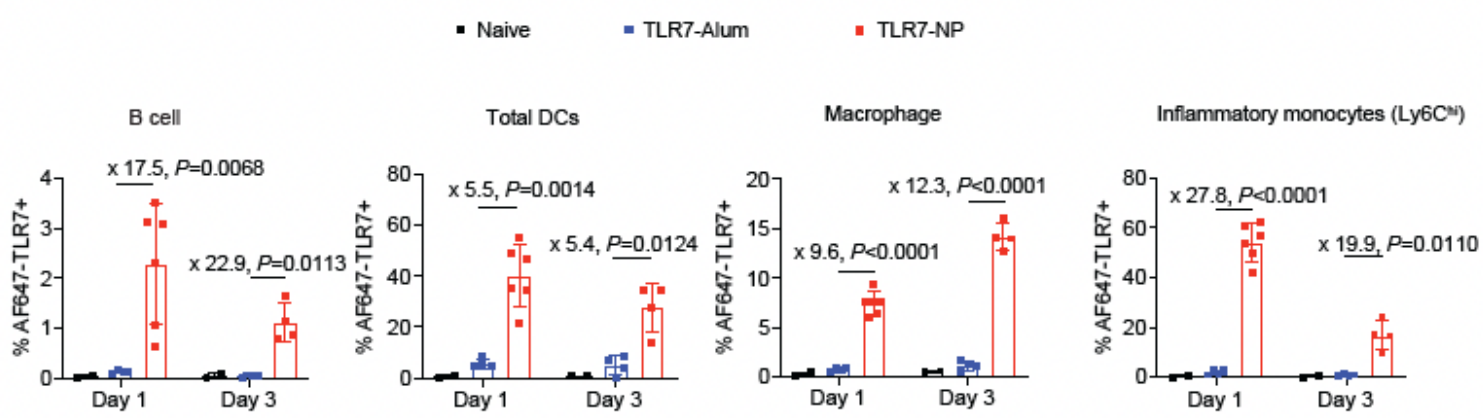

d
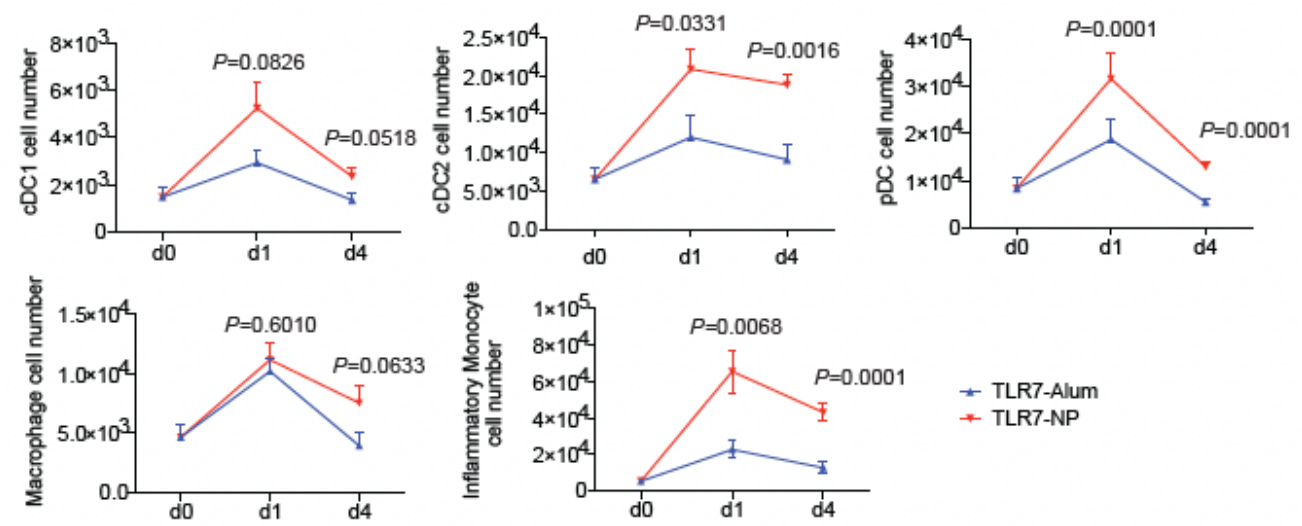

\section{Figure 2}

TLR7-NPs improve in vivo retention, draining lymph node (LN) accumulation, and cellular uptake of TLR7 agonist by antigen presenting cells, leading to persistent mobilization of DCs and cells of monocytic lineage in draining LNs. (a) AF647 (Ex/Em $=651 / 672 \mathrm{~nm}$ ) fluorophore-labeled gardiquimod $(20 \mathrm{nmol})$ were adsorbed on Alhydrogel (TLR7-Alum) or self-assembled into nanoparticles (TLR7-NPs), respectively, mixed with alum-adsorbed OVA antigen, and injected s.c. in C57BL/ 6 mice ( $\mathrm{n}=4$ mice per group from two 
independent experiments) followed by longitudinal whole-body fluorescence imaging. Shown are representative mice from each group and average normalized total radiance from groups of mice at the injection site over time. (b) Fluorescence imaging of excised draining LNs from C57BL/6 mice ( $\mathrm{n}=4$ mice per group from two independent experiments) at Day 1 and Day 3 post administration. Shown are two representative LN images and plotted mean fluorescence intensity (MFI) from one representative experiment. (c) C57BL/ 6 mice ( $n=6$ mice per group from two independent experiments) were immunized with AF647 labeled TLR7-Alum, or TLR7-NPs by s.c. injection, and flow cytometry was performed on draining LNs after Day 1 and Day 3 post immunization. (b) and (c) Bar graphs represent means \pm SD, respectively. (d) C57BL/ 6 mice ( $n=8-10$ from two independent experiments) were immunized with Alumadsorbed NP-OVA $(50 \mu \mathrm{g})$ plus TLR7 agonist $(20 \mu \mathrm{g})$ in either TLR7-Alum or TLR7-NPs on Day 0. Different types of innate immune cells were analyzed by flow cytometry for Day 0 (naïve mice) and Day 1 and 4 post immunization. Data are means $\pm S D$. Data are analyzed by unpaired $t$ test with Welch's correction. $P$ values are as shown. 
Figure 3:
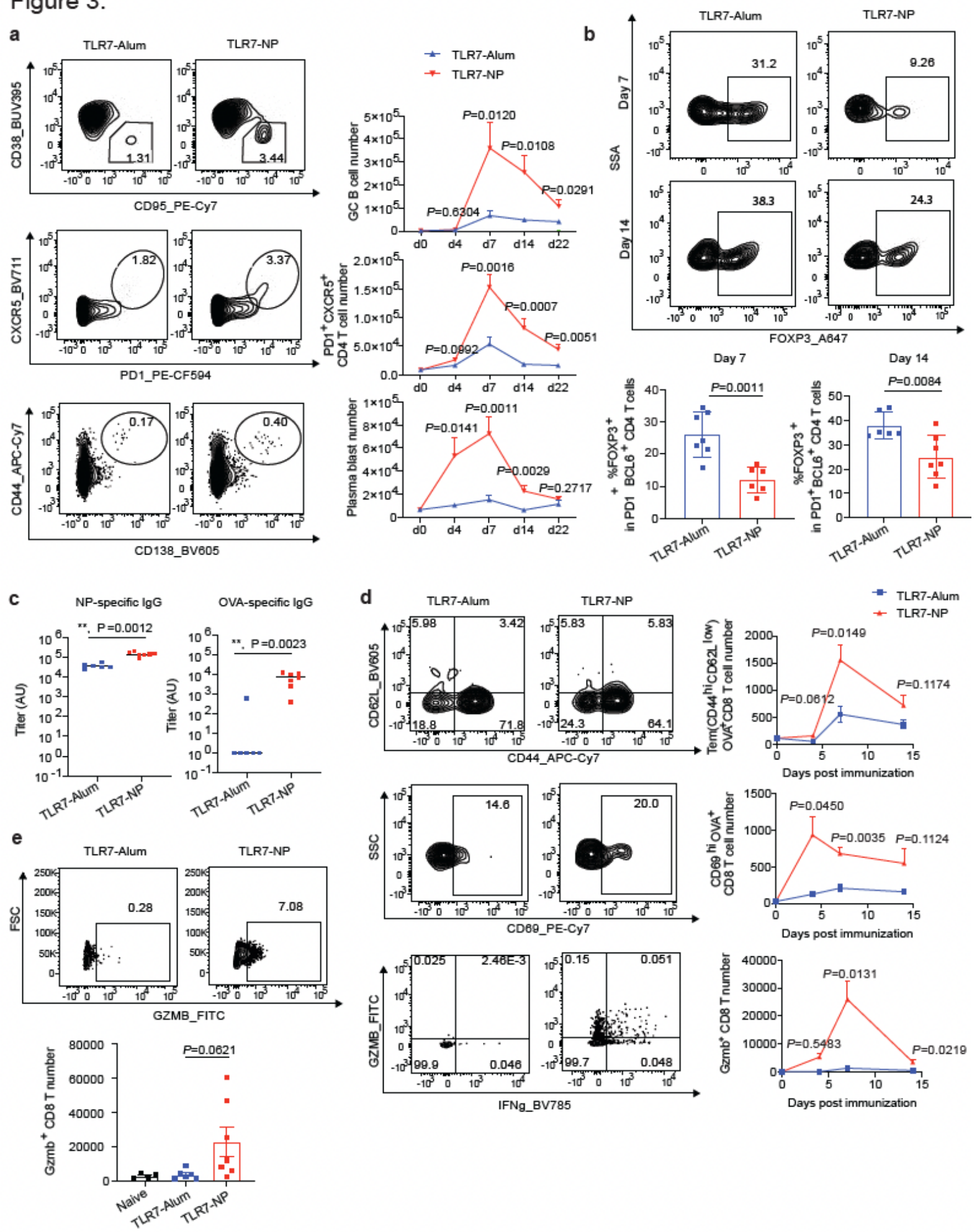

\section{Figure 3}

TLR7-NPs adjuvanted vaccine enhances the magnitude and quality of both antigen specific humoral and CD8+ T cell responses. C57BL/6 mice were immunized with Alum-adsorbed NP-OVA (50 $\mu$ g) plus TLR7 agonist $(20 \mu \mathrm{g})$ in either TLR7-Alum or TLR7-NPs on Day 0. (a) GC B cells, follicular CD4 T cell (CD4+CXCR5+PD1+) and plasma blasts in the draining LNs were analyzed by flow cytometry. Shown are representative flow cytometry plots of LN samples on Day 7 (left) and the cell number quantification of 
LN samples from Day 4, 7, 14 and 22. Data represent 6-8 mice from 2-3 independent experiments. (b) Follicular T cells (BCL6hiPD1hi) were analyzed for Tfr (FOXP3+) on Day 7. Shown are representative flow cytometry plots of LN samples on Day 7 and Day 14 (Top) and the cell number quantification of LN samples from Day 7 and 14 (Bottom). All the data are means \pm SD. (c) Serum samples collected 14 days post immunization were analyzed by ELISA for antibodies binding to immunodominant epitope (NP) and immunosubdominant epitope (OVA). (d) C57BL/6 mice ( $n=4-6$ per group) were immunized with Alumadsorbed NP-OVA $(50 \mu \mathrm{g})$ plus TLR7 agonist $(20 \mu \mathrm{g})$ in either TLR7-Alum or TLR7-NPs on Day 0. Shown are representative flow cytometry plots (left) and the corresponding quantification (right) of tetramerpositive effector memory (CD44hiCD62LlowOVA+) CD8+ T cells, tetramer-positive activated (CD69hiOVA+) CD8+ T cells, and functional CD8+T cells producing Gzmb in draining LNs on Day 4, 7 and 14. (e) C57BL/ 6 mice ( $\mathrm{n}=4$ per group) were immunized with Alum-adsorbed NP-OVA ( $50 \mu \mathrm{g})$ plus TLR7 agonist $(20 \mu \mathrm{g})$ in either TLR7-Alum or TLR7-NPs on Day 0 . Shown are representative flow cytometry plots (Top) and the corresponding quantification (Bottom) of functional CD8+T cells producing Gzmb in the lung on Day 14. Data are means \pm SEM. All the data are analyzed by unpaired $t$ test with Welch's correction. $P$ values are as shown. 
Figure 4:
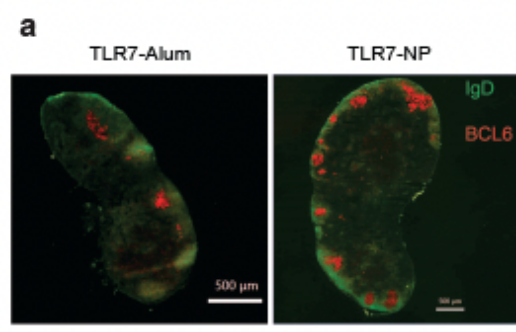

b

c

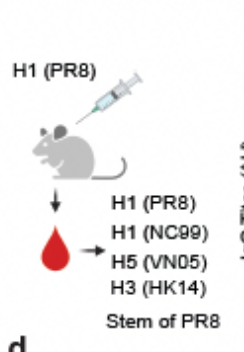

d

H1 (PR8)
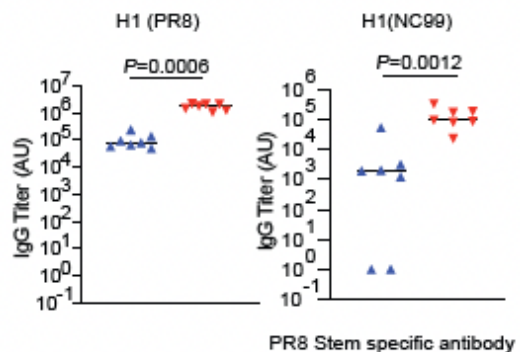

PR8 Stem specific antibody

H3 (HK68)
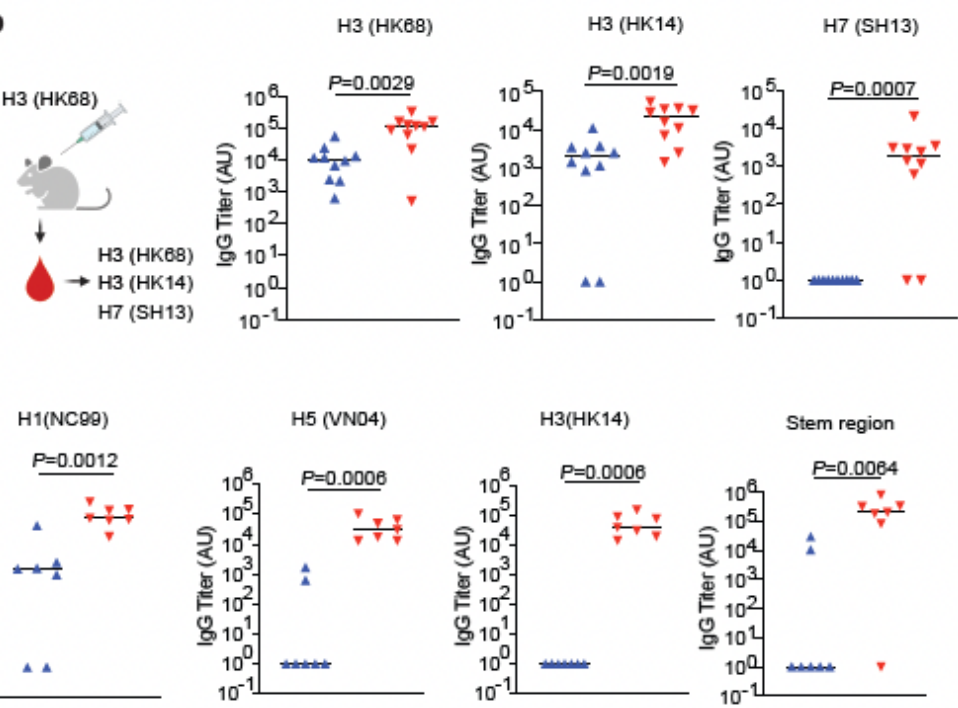

H3(HK14)
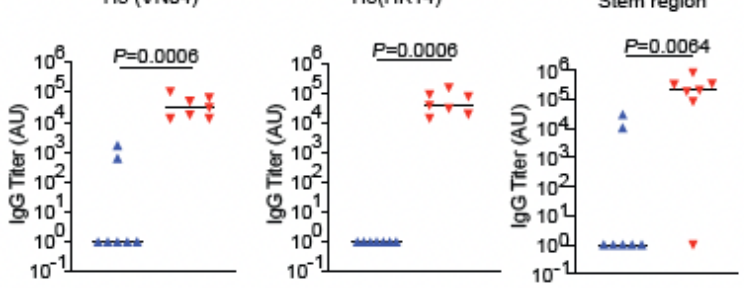

H1 (PR8)

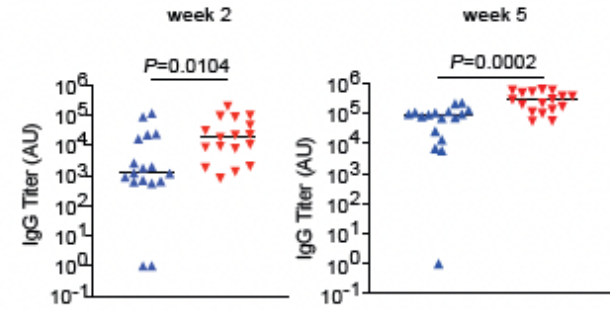

e
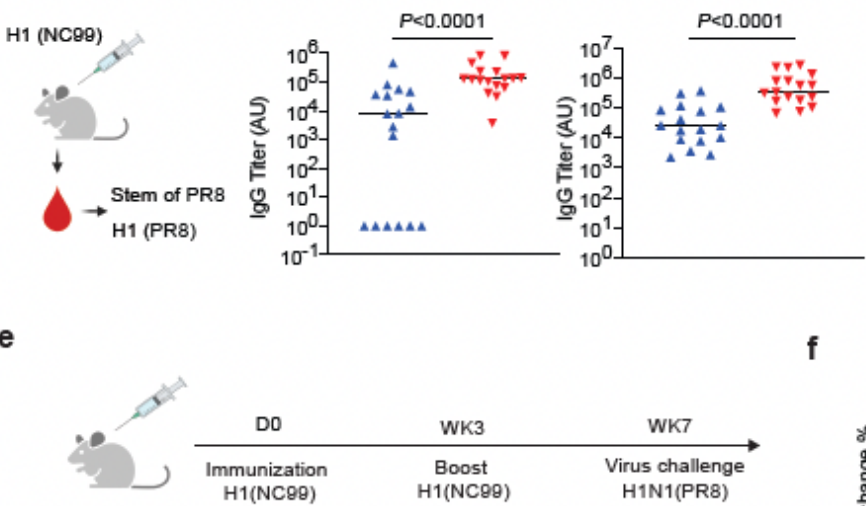

g

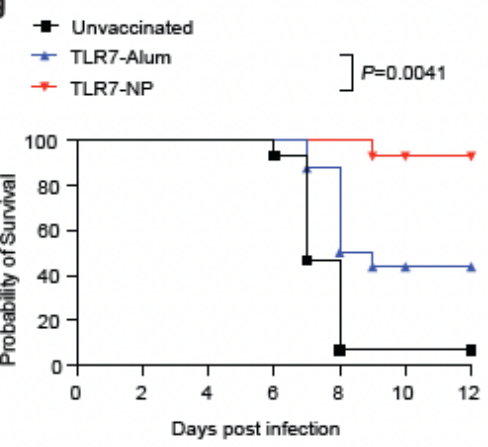

h
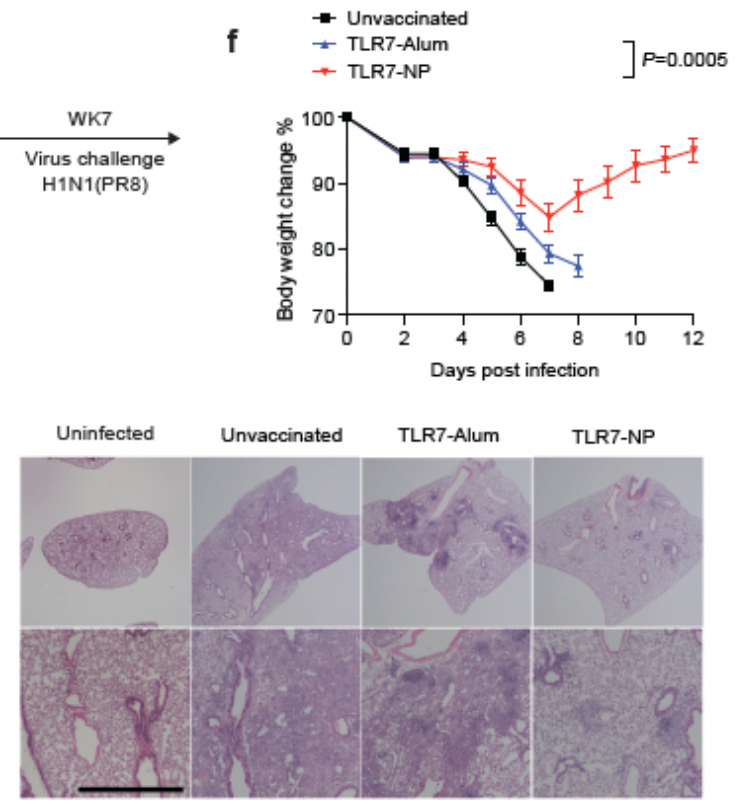

\section{Figure 4}

TLR7-NPs adjuvanted HA immunization efficiently elicits cross-reactive and stem binding antibodies and induces cross-protection from lethal heterosubtypic influenza virus challenge. (a) C57BL/6 mice were immunized with Alum-adsorbed HA of H1N1 A/Puerto Rico/8/34 (PR8 HA, $10 \mu \mathrm{g}$ ) plus TLR7 agonist (20 $\mu \mathrm{g}$ ) in either TLR7-Alum or TLR7-NPs at day 0. GC response in the draining LNs (Day 10) were analyzed by immunofluorescence. B cell zone and GC were stained with IgD and BCL6, respectively. Data show one 
representative LN imaging from two mice of each group. (b-d) Mice were immunized with Alum-adsorbed HA (10 $\mu \mathrm{g}$, strain as indicated) adjuvanted by TLR7 agonist $(20 \mu \mathrm{g})$ in TLR7-Alum or TLR7-NP. Serum samples collected two weeks (b-d) and five weeks (d) post immunization were analyzed by ELISA for antibodies binding to HAs from homo- and heterologous strains as indicated (b-d) and stem region of PR8 HA (c-d). All the data are medians with each dot representing one mouse. P values were calculated by Mann-Whitney Test. (e) Schematic illustration of vaccination and viral challenge schedule: C57BL/6 mice ( $n=14-16$ from two independent experiments) were immunized with Alum-adsorbed HA of H1N1 A/New Caledonia/20/1999 $(10 \mu \mathrm{g})$ plus TLR7 agonist $(20 \mu \mathrm{g})$ in either TLR7-Alum or TLR7-NPs on Day 0 (Prime) and Day 21 (Boost). Four weeks post second vaccine immunization, mice were infected with heterosubtypic H1N1 A/Puerto Rico/8/34 virus. The body weight changes ( $\mathrm{f}$ ) and survival (g) of mice corresponding to those described in (e). Data ( $f$ ) are analyzed by two-way ANOVA analysis. Data $(\mathrm{g})$ are analyzed by log-rank (Mantel-Cox) test. All P values are as shown. (h) Histological examination of the lungs from different groups of mice on Day 14 post infection (top 2X, bottom 8X; scale bar: $1000 \mu \mathrm{m}$ ). Data show one representative of at least four mice from each group. 
Figure 5:

a

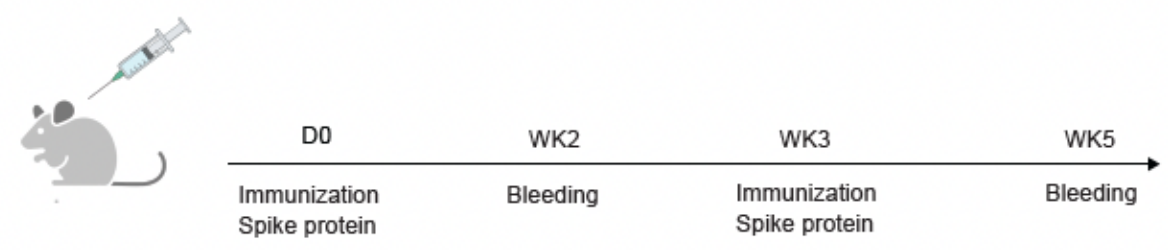

b

- TLR7-Alum

- tLR7-NP
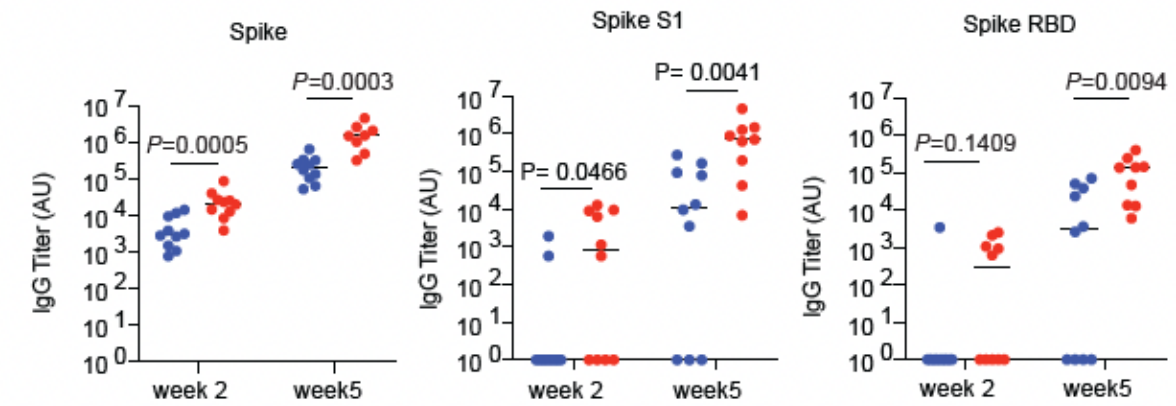

C

- TLR7-Alum

- TLR7-NP

RBD variant (N501Y from 3 lineages)

RBD variant (Brazil P.1)

RBD variant (India B.1.617)
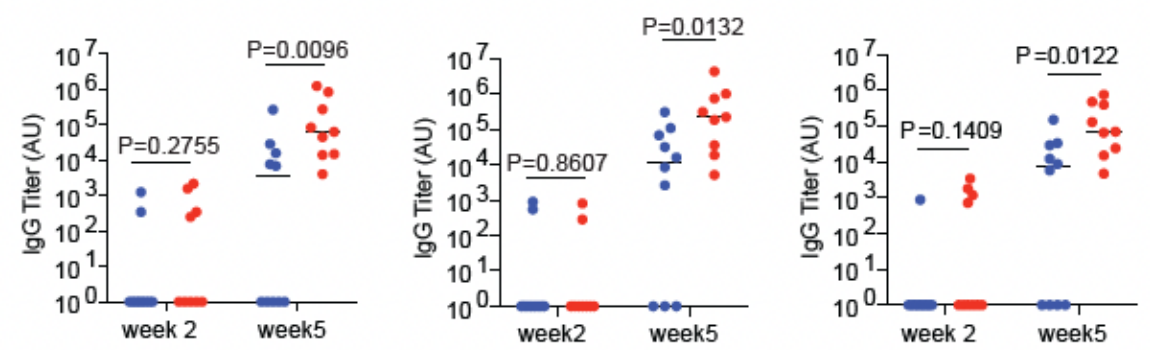

Indian delta-RBD

S1 variant (U.K. B.1.1.7)
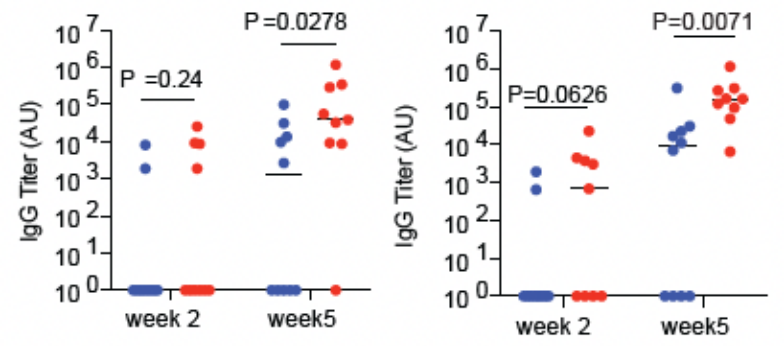

S1 variant (South Africa B.1.351)

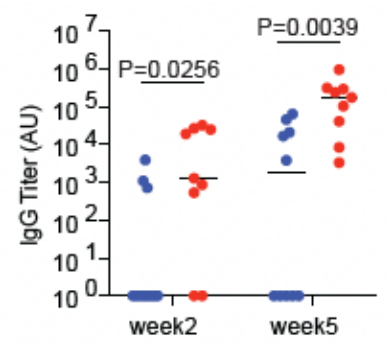

\section{Figure 5}

TLR7-NPs adjuvanted spike immunization induces cross-reactive antibodies against the SARS-CoV-2 variant lineages B.1.1.7, South Africa B.1.351, Brazil P.1 and Indian B.1.617. (a) C57BL/6 mice (8-10 mice for each group) were immunized with the Alum-adsorbed full-length SARS-CoV-2 Spike protein (5 $\mu \mathrm{g})$ plus TLR7 agonist $(20 \mu \mathrm{g})$ in either TLR7-Alum or TLR7-NPs at day 0. Serum samples were collected on Day 14 and Day 35 and analyzed by ELISA for antibodies binding to Spike RBD, Spike S1 and Spike (Wuhan 
reference strain) (b) and RBD or S1 from variants of all four lineages (c). All the data are medians with each dot representing one mouse. Data are analyzed by Mann-Whitney Test. P values are as shown.

Figure 6:

a

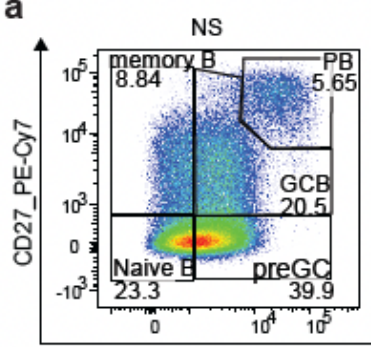

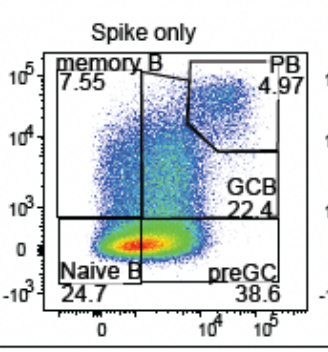

CD38_APC b

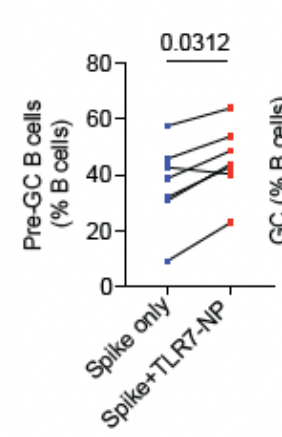

e

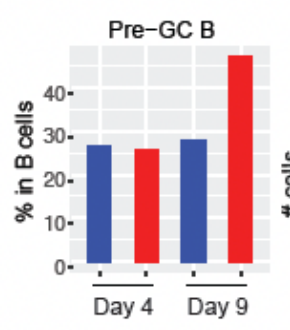

$\mathbf{f}$
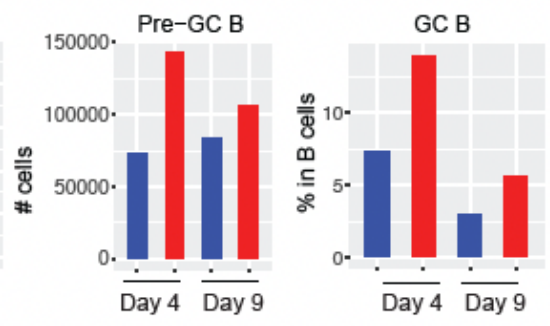

- RBD-NP

- RBD-NP+TLR7-NP

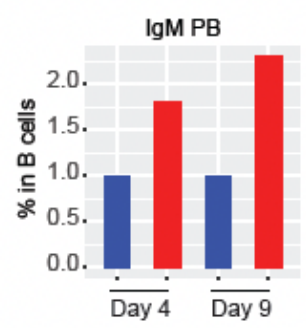

- RBD-NP
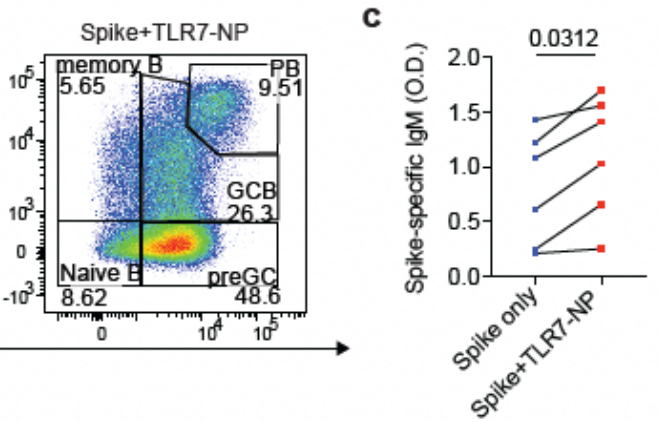

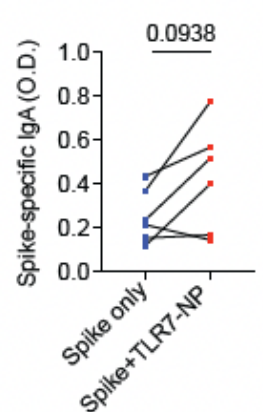

d

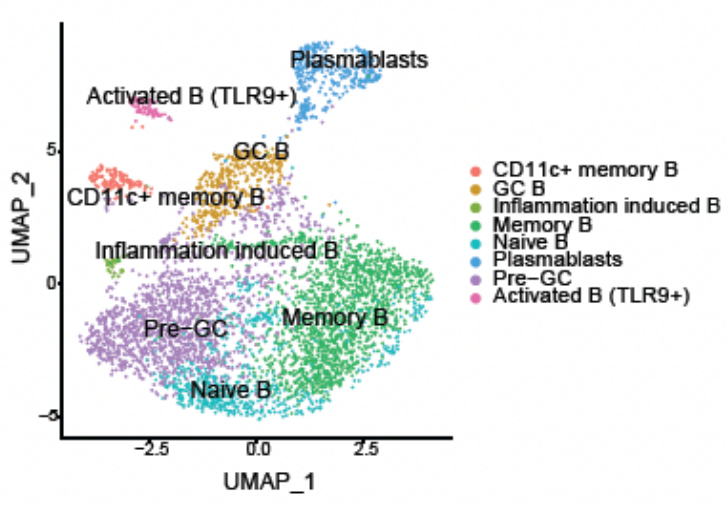

- RBD-NP+TLR7-NP
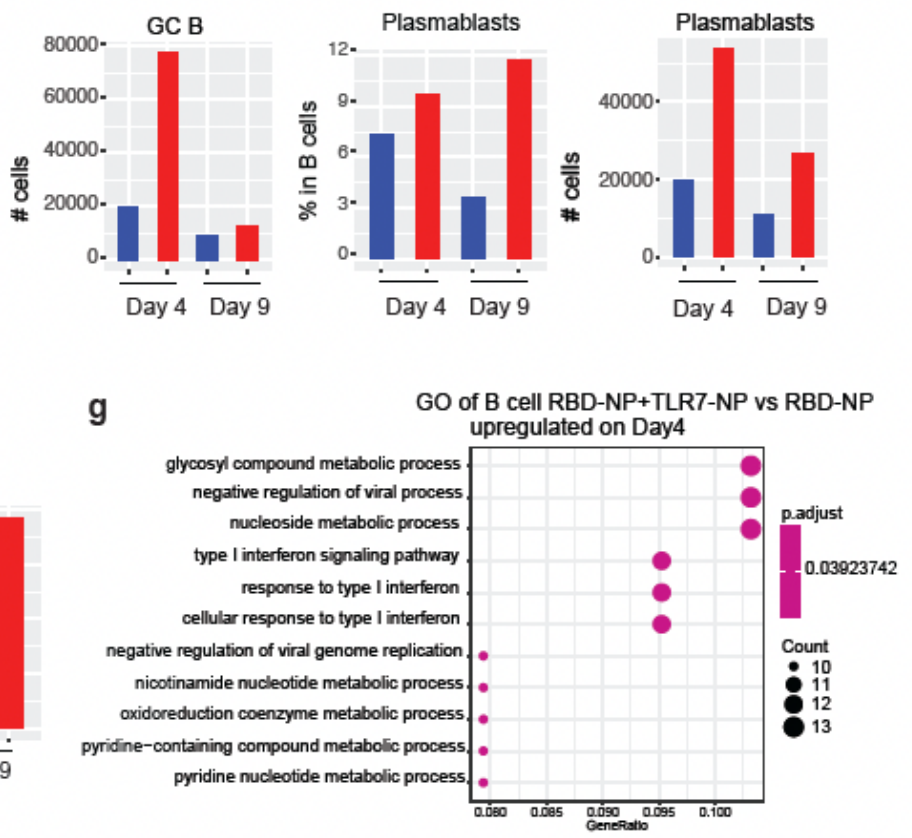

Figure 6

TLR7-NPs adjuvanted spike protein subunit vaccine elicits B cell differentiation and antibody response in human tonsil organoid cultures. (a) Representative flow cytometry staining of B cell phenotypes in unstimulated (NS), full-length spike protein only stimulated, and spike protein plus TLR7-NP stimulated 
organoid cultures from one donor on Day 14. Cells shown are pre-gated on total live B cells (CD45+CD19+CD3-). (b) Quantification of B cell differentiation towards pre-GC (CD38+CD27-), GC $(\mathrm{CD} 38+\mathrm{CD} 27+)$, and plasmablast (CD38+CD27++) in spike only stimulated, and spike plus TLR7-NPs stimulated organoid cultures ( $\mathrm{n}=7$ donors). (c) Quantification of spike-specific IgM and IgA from spike only stimulated and spike plus TLR7-NPs stimulated culture supernatants on Day 14 ( $n=6$ donors). P values shown were calculated by Wilcoxon matched-pairs signed rank test. (d) UMAP projection of tonsillar B cell scRNA-seq clusters. (e) Quantification of B cell differentiation subclusters (both frequency and total cell number in organoids) in scRNA-seq. ( $f$ ) Frequency quantification of plasmablast subclusters in scRNA-seq. (g) Gene ontologies for genes significantly upregulated in TLR7-NP plus antigen stimulated cultures versus antigen only stimulated cultures on day 4 .

\section{Supplementary Files}

This is a list of supplementary files associated with this preprint. Click to download.

- Supportinginformationfinal.pdf 